\title{
Heart Rate Dynamics with the Applications into a Quantitative Evaluation of Improvements on Cardiac Stress Endurance after High Intensity Interval Training in Healthy Men
}

\author{
Szi-Wen Chen ${ }^{1,2}$, Jiunn-Woei Liaw ${ }^{3}$, Burt Chang ${ }^{1}$ \\ ${ }^{1}$ Department of Electronic Engineering, Chang Gung University, Tao-Yuan, Taiwan \\ ${ }^{2}$ Neuroscience Research Center, Chang Gung Memorial Hospital, Linkou, Taiwan \\ ${ }^{3}$ Department of Mechanical Engineering, Chang Gung University, Tao-Yuan, Taiwan
}

\begin{abstract}
In this paper, we adopted a Cardiac Stress Index (CSI) to quantitatively evaluate improvements of cardiac stress endurance after high intensity interval training in healthy men. We measured the changes in CSI curves after a 5week cycling-based endurance training protocol that involved four repeated bouts of 4-minute high intensity effort followed by 3-minute active recovery. Seven healthy men ranging from 22 to 24 years old were recruited for undergoing the bipedal cycling endurance training. Each participant was asked to do ten-minute warm-up exercise to prevent sports injuries. We then incorporated the CSI into a cycling based cardiac monitoring system so the cardiac stress can be continuously and quantitatively assessed for the subjects undergoing the endurance training. The entire high intensity interval training process was performed three times a week and had lasted for five weeks. To conclude, numerical analysis results produced by the present study showed that CSI measures were reduced more after performing a 5-week endurance training compared with those obtained at the start phase of the training, indicating the cardiac stress endurance may be improved.
\end{abstract}

\section{Introduction}

There is evidence showing that high intensity interval training can be a time-efficient means for improving the health [1]. On the other hand, previous researches in literature have shown that a decreased in the fractal-like behavior of Heart Rate (HR) was detected during physical exercise [2-4]. In order to quantitatively evaluate and stratify the cardiac stress status, in a previous work we defined a time-varying parameter, called the Cardiac Stress Index (CSI), using the fractal HR dynamics in terms of the short-term Detrended Fluctuation Analysis (DFA) evaluated on the RR time series extracted from windowed Electrocardiogram (ECG) segments so the cardiac stress can be monitored and analyzed for subjects during the cycling exercise [4]. Results produced by the previous study indicated that CSI can be used as a means to continuously quantify the cardiac stress status [4].

Although previous research in literature indicated that brief duration bouts of exercise may effectively improve the maximal oxygen uptake and reduce blood pressure [1], there still remains a lack in direct evidence showing how the high intensity interval exercise may improve the cardiac stress endurance. This study aims to use CSI to achieve a quantitative evaluation of improvements on cardiac stress endurance after high intensity interval training in healthy men. Consequently, analysis results produced by our study showed that CSI measures were generally reduced more after performing a five-week endurance training compared with those obtained at the very beginning of the training, suggesting that the cardiac stress endurance may be improved.

\section{Methodology}

A submaximal testing was conducted in advance to determine the proper high intensity effort condition at $85 \%$ of maximal HR. Given a 20 -year-old young man, the $85 \%$ of maximal HR is $(220-20) \times 0.85=170 \mathrm{bpm}$ (beats per minute). This method allows the participant to output less physical effort with less risk. According to this pre-test, the personalized exercise performance was obtained to determine a proper condition for the high intensity effort parameter in the interval training test.

After the submaximal exercise test, the participant took at least one-day rest, and then was asked to perform a cycling exercise test with high intensity interval training (4-minute on and 3-minute off, four bouts). In addition, each participant was also asked to do ten-minute warm-up exercise before the test and five-minute cooling down after the test. As a result, it took 43-minute for experiment. In each cycle, a 4-minute on-loading was 
applied according to the result of the submaximal exercise test (i.e., $85 \%$ of maximal $\mathrm{HR}$ as described previously) and then followed by a 3-minute off-loading (a fixed lower power level). During the test, the ECG signal was recorded with $200 \mathrm{~Hz}$ sampling rate, and then the RR interval was obtained from these ECG profiles. Next, we used DFA to calculate the scaling exponent $\alpha$ [5]. The value of $\alpha$ represents the self-similarity of the detrended fluctuation of RR interval series. Normally, the value of $\alpha$ of a healthy one is larger than 1, indicating the HRV is highly correlated. If $0.5<\alpha<1$, the HRV is correlated. As $\alpha$ is close to 0.5 , it means that the time series is a random process. Therefore, we may see that the $\alpha$ value can reflect the cardiac self-regulation ability. In other words, $\alpha$ decreases as the cardiac stress increases.

In this study, we analyzed RR intervals to obtain the value of $\alpha$ per minute, which is the slope of DFA log-log plot [4]. Subsequently, we took the 3-point moving average of $\alpha$ to reduce the fluctuation level. According to a previous study, we have defined CSI to exhibit the cardiac stress status as [4]

$$
\text { CSI }=\frac{\text { Number } \text { of events with } \alpha \text { lower than } 1}{\text { Total number of events }}
$$

In (1), the CSI is an accumulated fractional function (a rational number), where the nominator is the number of the events of the $\alpha$ lower than the threshold (we set the threshold to 1.0) and the denominator is the total number of the events [4]. According to the definition, the CSI is the percentage of the occurrence of the events with $\alpha$ less than 1 during test and thus, the CSI is time-varying and its value is between 0 and 1 .

\section{Results and Discussion}

In this experiment, we recruited seven healthy young men ranging from 22 to 24 years old for participating the bipedal cycling endurance training program. Here, we incorporated the CSI technique into the cycling based cardiac monitoring system developed by ourselves so the cardiac stress can be continuously and quantitatively assessed for the subjects undergoing the endurance training. In our analysis, we measured the changes in CSI curves after a 5-week cycling-based endurance training protocol that involved four repeated bouts of 4-minute high intensity effort at $85 \%$ of maximal HR followed by 3 -minute active recovery at a fixed lower power level. The entire 43-minute high intensity interval training process was performed three times per week and had lasted for five weeks.

After the five-week training process, we then evaluated the change in cardiac stress endurance by assessing and the CSI values derived from all the participants during the test. Figure 1 provides an example of the averaged CSI values obtained after training in week 1 , week 3 , and week 5, respectively (from subject no. 6). It is revealed from Figure 1 that the averaged CSI value decreased as the training time increased.

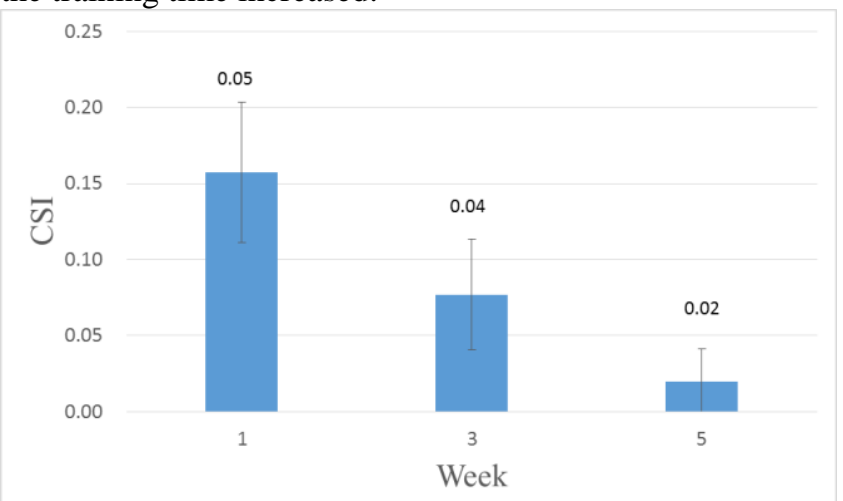

Figure 1. The average of CSI values obtained after training in week 1 , week 3 , and week 5 , respectively (from subject no. 6). It is revealed from the plot that the averaged CSI value decreased as the training time increased.

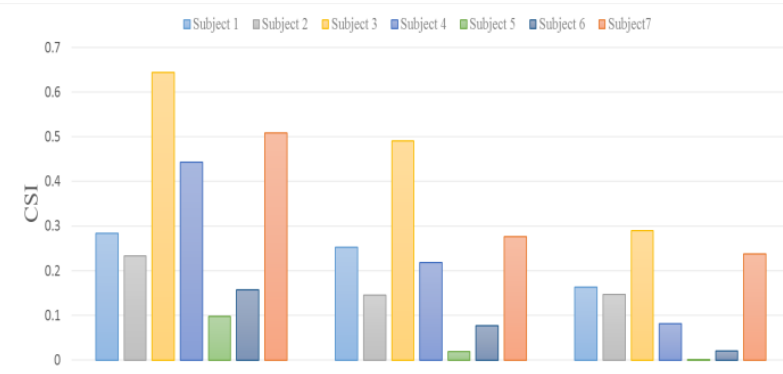

Figure 2. The average of CSI values derived from all the seven participants after training in week 1 , week 3 , and week 5 , as depicted in the left, middle and right portions, respectively in the plot. It is revealed from the plot that the averaged CSI value decreased as the training time increased for all the participants.

Furthermore, we also observed the CSI values obtained from all the participants during the entire training test. Figure 2 shows the averaged CSI values obtained after training in week 1 , week 3 , and week 5 , respectively, for all the seven participants. Similarly, we see from Figure 2 that the averaged CSI value also decreased as the training time increased for all the participants and thus, we may conclude that cardiac stress endurance might be effectively enhanced or improved after high intensity interval training.

\section{Conclusion}

Previous research in literature regarding the sports medicine indicated that high intensity interval training 
can be a time-efficient means for improving the health. In this study, we adopted a time-varying parameter, called the Cardiac Stress Index (CSI), defined based on the fractal HR dynamics in terms of the DFA of the RR time series to quantitatively evaluate and stratify the cardiac stress status. Our goal aims at using the CSI to achieve a quantitative evaluation of improvements on cardiac stress endurance after high intensity interval training in healthy men. We measured the changes in CSI curves after a 5week cycling-based endurance training protocol that involved four repeated bouts of 4-minute high intensity effort at $85 \%$ of maximal HR followed by 3 -minute active recovery at a fixed lower power level. The entire high intensity interval training process was performed three times per week and had lasted for five weeks. Consequently, numerical analysis results produced by this study indicated that CSI values were generally reduced more after performing a 5-week endurance training compared with those obtained at the beginning of the training, suggesting the cardiac stress endurance may be enhanced and thus improved.

\section{References}

[1] Tjønna AE, Leinan IM, Bartnes AT, Jenssen BM, Gibala MJ, Winett RA, Wisløff U. Low- and highvolume of intensive endurance training significantly improves maximal oxygen uptake after 10 -weeks of training in healthy men. PLoS ONE 2013; 8(5): e65382.

[2] Karavirta L, Tulppo MP, Laaksonen DE, Nyman K, Laukkanen RT, Kinnunen H, Häkkinen A, Häkkinen $\mathrm{K}$. Heart rate dynamics after combined endurance and strength training in older men. Med. Sci. Sport Exer. 2009; 41: 1436-43.

[3] Chen SW, Liaw JW, Chang YJ, L.-L Chuang LL, Chien CT. Combined heart rate variability and dynamic measures for quantitatively characterizing the cardiac stress status during cycling exercise. Computers in Biology and Medicine 2015; 63: 13342.

[4] Chen SW, Liaw JW, Chang YJ, Chan HL, Chiu LY. A cycling movement based system for real-time fatigue and cardiac stress monitoring and analysis. PLoS ONE 2015; 10(6): e0130798.

[5] Peng CK, Havlin S, Stanley HE, Goldberger AL. Quantification of scaling exponents and crossover phenomena in nonstationary heartbeat time series. Chaos 1995; 5(1): 82-86.

Address for correspondence.

Szi-Wen Chen

Department of Electronic Engineering, Chang-Gung University

259 Wen-Hwa $1^{\text {st }}$ Rd., Tao-Yuan, Taiwan

chensw@mail.cgu.edu.tw. 
Page 4 